\title{
Effects of the Transcription Factor Ace2 from Trichoderma Reesei on Cellulase and Hemicellulase Expression in Trichoderma Orientalis EU7-22
}

Yuyu Li

Xiamen University

Yong Xue

Xiamen University

Jian Liu ( jianliu@xmu.edu.cn )

Xiamen University https://orcid.org/0000-0003-0696-0793

Lihui Gan

Xiamen University

Minnan Long

Xiamen University

Original Article

Keywords: transcription factor, Ace2, Trichoderma reesei, cellulase, hemicellulase, Trichoderma orientalis

Posted Date: February 2nd, 2021

DOI: https://doi.org/10.21203/rs.3.rs-164454/v1

License: (c) (i) This work is licensed under a Creative Commons Attribution 4.0 International License.

Read Full License

Version of Record: A version of this preprint was published at Applied Biochemistry and Biotechnology on February 19th, 2021. See the published version at https://doi.org/10.1007/s12010-021-03529-5. 


\section{Abstract}

Trichoderma orientalis (T. orientalis) EU7-22 has a complete cellulase system and shows a remarkable enzyme activity with high potential in the industry. Ace2 is an important transcriptional factor for cellulase and hemicellulase expression in Trichoderma reesei (T. reesei). However, the ace2 gene can not be found in the genome of $T$. orientalis. Researches show that the mechanism of cellulase transcriptional regulation in $T$. orientalis keeps high similarity with $T$. reesei up till now. So, in this study, the ace 2 of Trichoderma reesei QM9414 was heterologous expressed in T. orientalis EU7-22. As a result, xylanase activity and $\beta$-glucosidase activity of ace 2 heterogeneous expression strains are improved and total cellulase activity is decreased. The result of qPCR is in accordance with enzyme activities. This study provides a reference for an in-depth study on transcriptional regulation mechanisms of $T$. orientalis.

\section{Introduction}

As a renewable resource, lignocellulosic biomass, which typically consists of cellulose, hemicellulose and lignin, are plentiful and widely distributed on the earth. Cellulose and hemicellulose, as the main components of leaves, stalks and wood of plants, could become a substitute for the starch if suitably treated for making because they can be degraded to fermentable sugars, e.g. oligosaccharides, glucose, xylose, etc. So the bio-ethanol from lignocellulosic feedstocks is called "second generation" [1]. However, the cost and the efficiency of the enzymes capable of breaking cellulose down has always been a problem of the biofuel industry. Nowadays, the most popular enzyme producer is which can produce whole cellulase system, including endoglucanase (EG, EC 3.2.1.4), cellobiohydrolase (CBH, EC 3.2.1.91), $\beta$-glucosidase (BG, EC 3.2.1.21), lytic polysaccharide and so on[3, 4].

To enhance the efficiency of cellulase production, plenty of methods have been tried, including sitedirected mutagenesis, transcriptional regulation factors engineering, etc. $([5,6])$. In this study, EU7-22 is reserved in our lab and obtained from $T$. orientalis XC-9 by a complex mutation $[7,8]$. It keeps high similarity with $T$. reesei. By comparing the ability to produce different cellulase of EU7-22 with that of other common strains producing cellulase used in the industry, Long concluded that EU7-22 has a complete cellulase system and shows a remarkable enzyme activity with high potential in the industry[8]. Transcriptional regulation of filamentous fungi represents a logical engineering target to improve cellulase production. Researches on transcriptional regulation factors have been launched widely[3]. A suite of transcriptional regulation factors plays an important role in the production of cellulase in fungi. Some transcriptional factors regulating the expression of cellulase- and xylanase-encoding genes have also been identified in EU7-22. In T. reesei, it is known that the obvious difference of cellulase production between the more efficient strain Rut-C30 and the less efficient strain QM9414 is the truncation of Cre1. Cre1 shuts down the transcription of its target genes when glucose exists [9]. In EU7-22, Cre1 is also one of the master regulators which is a $\mathrm{C} 2 \mathrm{H} 2$ type transcription factor and participates in carbon catabolite repression. The Cre1 disrupted strain shows both a higher basal level and a higher induced level of cellulase production[10]. Another typical repressor of cellulase expression is Ace1 [11] , and deletion of ace1 increased the expression of all the crucial cellulase genes and two xylanase genes in the certain 
induced cultures. In the basic research, it is also represented as a repressor in EU7-22. In addition to transcriptional repressor Cre1 and Ace1, some transcriptional activators have also been studied, such as Ace3 and Xyr1 [7, 12-14]. It has been proven that overexpression of Xyr1 leads to a higher expression of cellulases and abolishes their catabolite repression in the presence of glucose. Besides, when two important transcription activators Xyr1 and Ace3 were overexpressed in the EU7-22 strain, the cellulase production was greatly improved[7]. In Ace3 as a Zn(II)2Cys6 type transcriptional factor binds the promoters of many cellulase genes, the cellulose response transporter gene crt 1 and transcription factorencoding genes, including $x y r 7[12]$. Xyr 1 is also a $\mathrm{Zn}(\mathrm{II}) 2 \mathrm{Cys} 6$ type transcriptional activator, and it binds to a GGCTAA motif arranged as an inverted repeat in the xyn1 promoter [15]. They are both responsible for the activation of lots of genes involved in the degradation of cellulose and xylan. By comparing the DNA sequence of QM9414, T. orientalis EU7-22 keeps high similarity with its cellulase transcriptional regulators as well[16].

However, Ace2 is one of the most special cellulase transcriptional regulators existing in $T$. reesei but it cannot be found in T. orientalis. The N-terminal part of the deduced Ace2 protein has a typical zinc binuclear cluster DNA-binding domain of the fungal type (Zn(II)2Cys6), first characterized in $S$. cerevisiae[17]. In T. reesei, Aro. $\mathrm{N}$ et al. indicated that Ace2 binds in vitro to the GGCTAATAA site in the $c b h 1$ promoter. This site also contains the proposed binding sequence of the xylanase activator XInR from Aspergillus niger[18]. By knocking out ace2 in T. reesei strain ALKO2221, the cellulase and hemicellulase activities both drastically decreased with reduced transcript levels of lignocellulolytic enzymes. Stricker also found that both Xyr1 and Ace2 can bind the complete motif of Xyn2 promoter consisting of a GGGTAA-element and a CCAGCC-element. The $\triangle$ ace2-strain showed faster initial inducibility when induced by xylan and xylobiose although final levels of xylanase activity of the parental strain were higher[19]. Some relevant studies were given by Xiong et al. They have successfully transformed the Ace2 transcription factor encoding gene in $T$. reesei and gotten five genetically engineered strains. Among which, the result displayed that only one has exhibited a significant increase in enzyme activity, but the other four showed lower total cellulase activities than the parental strain $T$. reesei QM9414[20]. Also, Ace2 was not subject to carbon catabolite repression, because in the $\triangle$ cre1 strain no increased expression levels of ace2 gene were observed.[21]

In this study, on considering the high similarity between these two fungi, Ace2, as a cellulase transcriptional activator, may contribute to the promotion of cellulase production if introduced to EU7-22. However, the result of heterologous expressed ace2 in EU7-22 is beyond expectation. Some of the cellulase and hemicellulase activities increased comparing to the parent strain, but some of the enzymic activities did not increase, even decreased. It provides the potential in the applications for differentiated purposes. This study reflected the complexity of mechanisms of regulation in vivo and interspecific difference between $T$. reesei and $T$. orientalis.

\section{Materials And Methods}

2.1. Microorganism strains 
T. reesei QM9414 (ATCC 26921) was used for cloning ace2 gene, promoter (Peno) and terminator (Teno) of eno gene. T. orientalis EU7-22 was used as the parental strain for transformation throughout this study.

\subsection{Culture media}

Minimum medium (MM medium) is used for strain growth consisting of $20.0 \mathrm{~g} / \mathrm{L}$ glucose, $15.0 \mathrm{~g} / \mathrm{L}$ $\mathrm{KH}_{2} \mathrm{PO}_{4}, 5.0 \mathrm{~g} / \mathrm{L}\left(\mathrm{NH}_{4}\right)_{2} \mathrm{SO}_{4}, 0.6 \mathrm{~g} / \mathrm{L} \mathrm{CaCl}, 0.6 \mathrm{~g} / \mathrm{L} \mathrm{MgSO}{ }_{4}$ and $10 \mathrm{~mL} / \mathrm{L}$ trace element solution (The solution containing $0.2 \mathrm{~g} / \mathrm{L} \mathrm{CoCl}_{2}, 0.5 \mathrm{~g} / \mathrm{L} \mathrm{FeSO}_{4} \cdot 7 \mathrm{H}_{2} \mathrm{O}, 0.14 \mathrm{~g} / \mathrm{L} \mathrm{ZnSO}_{4} \cdot 7 \mathrm{H}_{2} \mathrm{O}$ and $\left.0.16 \mathrm{~g} / \mathrm{L} \mathrm{MnSO}{ }_{4} \cdot \mathrm{H}_{2} \mathrm{O}\right)$. The $\mathrm{pH}$ of $\mathrm{MM}$ medium is 4.8 . The solid MM medium(SMM medium) is $\mathrm{MM}$ medium adding with $2 \%$ agar. The SMM medium supplemented with $44.7 \mathrm{~g} / \mathrm{L} \mathrm{KCl}$ is the $\mathrm{MK}$ medium which is used in the process of protoplast regeneration. The medium for growth observation is SMM medium with different carbon sources, including avicel (MA), cellobiose(MB), CMC-Na (MC), glycerol(MG), lactose(ML), xylose (MX) and xylan from bagasse (MY). The strain was cultured in an induction medium (ABP medium) at $\mathrm{pH} 5.2$ for cellulase production containing $10.0 \mathrm{~g} / \mathrm{L}$ wheat bran, $20 \mathrm{~g} / \mathrm{L}$ avicel, $5.0 \mathrm{~g} / \mathrm{L}$ peptone, $2.5 \mathrm{~g} / \mathrm{L} \mathrm{KH}_{2} \mathrm{PO}_{4}, 0.5$ $\mathrm{g} / \mathrm{L} \mathrm{CaCl} 2,0.5 \mathrm{~g} / \mathrm{L} \mathrm{MgSO}_{4}$ and $10 \mathrm{~mL} / \mathrm{L}$ trace element solution.

\subsection{Construction of ace2 expression cassette}

The heterogeneous expression of ace 2 cassette was constructed as follows. The primers PA2eno-1 and PA2eno-3, as well as TA2eno-4 and TA2eno-5 were used to amplify Peno and Teno from T. reesei QM9414 genomic DNA. Ace2 gene (GenBank accession no. CP016233.1) using primers ace2-F and ace2-R was also amplified from $T$. reesei QM9414 genomic DNA by PCR. The hygromycin resistance cassette was cloned from pUR5750 plasmid containing hygromycin resistance gene (hygromycin phosphotransferase, $h p h$ ) which was preserved in our laboratory using primers PgpdA-hph-F and hph-TgtrpC-R. Then, the ace2 expression cassette could be obtained by fusion PCR with primers PA2eno-2 and TgtrpC-2 using the fragments mentioned above as the templates[22]. After that, primer hph-F and hph-R were used to verify the existence of the hygromycin resistance in the transformants, and then they were chosen after $96 \mathrm{~h}$ fermentation under the inducing condition of ABP medium. Sequences of all primers are given in Table1.

The DNA polymerases used in this study includes PrimeSTAR® HS DNA Polymerase (Takara, Japan) for amplifying 1-2kb gene segments, PrimeSTAR ${ }^{\circledR}$ Max DNA Polymerase (Takara, Japan) for synthesizing 3kb-10kb gene segments, and the 2xTaq Plus Master Mix (Dye Plus) (Vazyme, China) for the PCR of verifying transformants.

\section{Table 1. Sequences of all primers}




\begin{tabular}{|ll|}
\hline Primers & Sequencel5'-3' 1 \\
\hline PA2eno-1 & GGAGGGATATAGACTATGGCTCATG \\
\hline PA2eno-2 & GTGATTCCGTCCTGGATTGC \\
\hline PA2eno-3 & CATGCTTGCCGGAGGTCCATTTTGAAGCTATTTCAGGTGGCTG \\
\hline ace2-F & ATGGACCTCCGGCAAGCAT \\
\hline ace2-R & TCACTTCAGCAGTCTGGCACTG \\
\hline TA2eno-4 & CAGTGCCAGACTGCTGAAGTGAATGGCCACGAGAGACAACTACCTAT \\
\hline TA2eno-5 & GATGGCGTCGTTGATGTTTCG \\
\hline PgpdA-hph-F & CGAAACATCAACGACGCCATCGATCTTTCGACACTGAAATACGTC \\
\hline hph-TgtrpC-R & AAGAAGGATTACCTCTAAACAAGTG \\
\hline TgtrpC-2 & GTGCATTCTGGGTAAACGACTC \\
\hline hph-F & GAATCGGTCACTACACTACATGGC \\
\hline hph-R & CGCCCAAGCTGCATCATCGAA \\
\hline
\end{tabular}

\subsection{Fungal transformation}

The method of protoplast transformation (obtaining the transformants) follows Xue et al [7].

\subsection{Measurement of enzyme activity}

in $\mathrm{MM}$ medium. Then after $18 \mathrm{~h}, 5 \%$ of the culture was incubateded into ABP culture for fermentation. The fermentation broth was collected by centrifugation every $24 \mathrm{~h}$ till $120 \mathrm{~h}$. The FPase, CMCase, pNPCase, pNPGase, xylanase and pNPXase were measured and they represent the total cellulase, endoglucanases, exoglucanases, $\beta$-glucosidase, xylanase and xylosidase activity, respectively [23]. The definition of one unit of enzyme activity follows the report [7].

\subsection{Measurement of total soluble protein}

Total soluble protein was measured as the manufactory's instructions indicated by a Modified Bradford Protein Assay Kit (Sangon, China).

\subsection{RNA-extraction, cDNA-synthesis, and real-time PCR}

RNA samples from transformant and parental strain for analyses of gene expression were prepared from mycelium powder obtained by grinding the filtrated biomass from shake flasks in liquid nitrogen. Then samples were lysed and cleaned up by using TaKaRa MiniBEST Universal RNA Extraction Kit (TaKaRa, Japan). In the procedure of isolation of total RNA, the materials pipette tips and columns are dealt with diethylpyrocarbonate treated water and the mortars are dry-heat sterilized at $180^{\circ} \mathrm{C}$ for 60 min to ensure 
there is no RNA lytic enzyme. Then 5× All-In-One MasterMix Kit (Vazyme, China) is used for synthesizing cDNA (reverse transcription).

The primers for amplifying genes encoding cellulases and regulatory factors used for real-time quantitative PCR are listed in Table 2.

\section{Table 2. The primers for qPCR}




\begin{tabular}{|c|c|}
\hline Primers & Sequence $\left[5^{\prime}-3^{\prime}[]\right.$ \\
\hline actin-YGF & TCCATCATGAAGTGCGAC \\
\hline actin-YGR & GTAGAAGGAGCAAGAGCAGTG \\
\hline cbh I-YGF & ATCGGCTTCGTCACGCAATC \\
\hline cbh I-YGR & ACGCCACCATCCGCATCCA \\
\hline cbh II-YGF & GACAAACCTCGGCACTCC \\
\hline cbh II-YGR & GACCAGCGTCCAGATACATT \\
\hline eg I-YGF & CAGGGCTTCTGCTGTAATGAG \\
\hline eg I-YGR & TTG AACTGGGTGATGATGGTG \\
\hline eg II-YGF & GCTCCGCCAGAATAACCG \\
\hline eg II-YGR & CAGCCA ACATAGCCAAGATAGAC \\
\hline bgl 1-YGF & ATCACCTACCCGCCTTCA \\
\hline bgl 1-YGR & TCTCGTCGTCGGATGTTG \\
\hline xyn I-YGF & CGTCAACACGGCGAACCA \\
\hline xyn I-YGR & CGGTGATGGAAGCAGAGCC \\
\hline bxl1-YGF & TGTTCGCACAAGCAACGCT \\
\hline bxl1-YGR & ACTCGTCGGTGTTCAAGGCT \\
\hline xyr1-YGF & TGCTTGACGACGACGACTTGT \\
\hline xyr1-YGR & ACGCCGTAGAAGAAGGGCAT \\
\hline cre1-YGF & TCTACGGCTCCTTCTTCTC \\
\hline cre1-YGR & ACAAGTTCCTCAGACTCGG \\
\hline ace1-YGF & TGATGAGGGCTTTGACGAGTC \\
\hline ace1-YGR & GGTTGAAGATGTCGGGCTGT \\
\hline ace2-YGR & GACAAGAAGCTCAGGTGTC \\
\hline ace2-YGF & ACTGTGTTCATGGCTGTG \\
\hline ace3-YGF & TGCTGAGGGTGATGAACGAG \\
\hline ace3-YGR & GGGTGAATCCTGGTTGCGAT \\
\hline
\end{tabular}

Quantitative PCR was performed on fluorescence quantitative thermocycler ( $A B I, U S A)$. Amplification reactions were performed using the Universal SYBR qPCR Master Mix (Vazyme, China). Data analysis 
was performed using the $2^{-\Delta \Delta C T}$ method. As a reference gene, we used actin which shows stable transcript levels across different conditions [24].

The RT-qPCR protocols are initially denatured at $95^{\circ} \mathrm{C}$, followed by 45 cycles of $15 \mathrm{~s}$ at $95^{\circ} \mathrm{C}, 15 \mathrm{~s}$ at $59^{\circ} \mathrm{C}$ and $15 \mathrm{~s}$ at $72^{\circ} \mathrm{C}$. All reactions were repeated for three times in $20 \mu \mathrm{L}$-mixtures including $1 \mu \mathrm{L}$ forward primer, $1 \mu \mathrm{L}$ reverse primer, $1 \mu \mathrm{L} \mathrm{CDNA(10-fold} \mathrm{diluted)} \mathrm{as} \mathrm{a} \mathrm{template} \mathrm{and} 10 \mu \mathrm{L}$ Universal SYBR qPCR Master Mix. Each run included a blank (sterile bi-distilled water instead of the sample). All samples were analyzed in three independent biological experiments with three RT-qPCR replicates in each assay.

\section{Results And Discussion}

\subsection{Construction of ace2 recombinant transformants}

The ace 2 cassette has been successfully synthesized by fusion PCR. The four gene segments were amplified as previously described and shown in Fig. 1. The lanes1-4 represent the Peno (1669 bp), ace2 (1026 bp), Teno (986 bp) and hygromycin resistance cassette (3936 bp) respectively. According to Li et al., the promoters of enolase (eno) were found under the condition of glucose while the transcript levels of the gene was much higher than others[25].

\subsection{Screening of recombinant transformants}

The strains were screened out from the of PDA medium with $100 \mu \mathrm{g} / \mathrm{mL}$ hygromycin B and $0.01 \%$ tritonX -100. The $h p h$ gene of the screening strains was amplified with the primer $h p h-F$ and $h p h-R$, and 9 recombinant strains were obtained through 4 times of transforming operation named 0-1, 0-2, 0-3, 0-4, 0$5,1-1,1-2,2-9$ and 3-1. The highest enzyme activity of EU7-22 was judged at the fourth-day of fermentation. FPA activity were quantitated as $0.86,0.97,1.08,1.01,0.51,0.88,1.00,0.93$ and 0.93 $\mathrm{IU} / \mathrm{mL}$, and the total cellulase activity all decreased compared with the parental strain $(1.15 \mathrm{IU} / \mathrm{mL})$ after the fermentation for 4 days. Three strains (0-2, 1-1 and 2-9) with an average performance of total cellulase activity were selected for further study.

\subsection{Enzyme activity of transformants and total}

According to Fig. 4, in ABP inducing medium, the FPase activities of the ace2 heterologous expression strain were lower than that of the parental strain, and the recombinant strains had reductions by $4.27 \%$, $13.06 \%$, and $6.65 \%$, respectively. By measuring the CMCase activity, it is noticed that the expression of ace2 gene reduced the expression of endocellulase activity of $T$. orientalis, and the endocellulase activities reduced by $7.01 \%, 21.50 \%$, and $20.14 \%$, respectively. The CBH activity of the $0-2$ strain increased by $20.69 \%$, and the enzyme activities of $1-1$ and 2-9 were basically the same as the original strain.

$\beta$-glucosidase activity was improved, and the enzyme activities of the three strains (0-2, 1-1 and 2-9) increased by $45.81 \%, 28.40 \%$ and $20.13 \%$. The activity of xylanase was also improved, and the enzyme activities of the three strains (0-2, 1-1 and $2-9)$ increased by $23.71 \%, 7.74 \%$ and $10.70 \%$. The $\beta$-xylosidase 
activity. The $\beta$-xylosidase activity of three strains (0-2, 1-1 and 2-9) reached their peak values on the third day, decreased by $38.91 \%, 17.01 \%$, and $56.08 \%$, respectively. These results indicated that the expression of ace 2 in $T$. orientalis largely improved the $\beta$-glucosidase activity and xylanase activity. In $T$. reesei, deletion of ace 2 gene resulted in reduced expression levels of cellulases on Solka floc cellulose[18, 19] and overexpression of ace2 gene exhibited significant increase in [20]. However, in contrast with the claim of Ace2 activating the cellulase production, most of the cellulase activities decreased after expressing ace2 in EU7-22. Based on ace2 expression, the species differences between T. reesei and T. orientalis are significant.

The extracellular protein of strain (0-2, 1-1 and 2-9) reduced by $14.32 \%, 21.5 \%$ and $8.5 \%$ when compared with the parent strain EU7-22. It kept consistent with the result of decreasing.

\subsection{Quantitate PCR analysis}

After cultivation on the solid PDA and MM medium, there is a significant difference in growth phenotype between 0-2 and EU7-22. Considering that the insertion site may have an impact on the of 0-2, so 1-1 was chosen for detailed research later.

The result indicated the ace2 transcript can be detected in the strain 1-1 but absent in EU7-22. The $\triangle \mathrm{CT}$ of ace 2 is 2.41 compared to the CT value of the actin gene. This demonstrated that ace 2 was successfully expressed in the recombinant strain 1-1. The genes encoding cellulase and hemicellulase in the strain 1-1 were not significantly increased(Fig. 6). The value of $2^{-\triangle \Delta C T}$ of $b g / 1$ is 1.20 and xyn1 is 1.79 , which is kept in line with the cellulase activities described before. The level of $x y n 2$ transcription is too low to be detected.

The values of $2^{-\triangle \triangle C T}$ of $c r e 1$, ace 1 , ace 3 and $x y r 1$ is $0.54,0.73,0.57$ and 0.37 , respectively. According to the result of qPCR, we provide the evidence that ace 2 has a certain role in the induction of the major cellulase and xylanase genes of EU7-22. The transcriptional quantity of cre1 and ace 1 encoding cellulase transcription repressor Cre1 and Ace1 decreased. The amount of ace 3 and $x y r 1$ transcription of 1-1 was decreased. According to the lost enzyme activities of the ace3 disrupted strain in EU7-22[26], it can be deduced that in ace2 expression strain 1-1, Ace3 plays an influential role.

\subsection{Phenotype and growth rate analysis}

The result demonstrated that expression of ace2 in EU7-22 scarcely affected the conidia formation after cultured for 72 h (Fig. 7a).

The strain 1-1 spread slower than EU7-22 on all medium except on MB(cellobiose) and MX(xylose) which coincided with the result of increased $\beta$-glucosidase activity and xylanase activity (Fig. 7b). It can be deduced that the decreased total caused the slower speed of the utilizing avicel.

\section{Conclusions}


In conclusion, Ace 2 is responsible for the change of cellulase activities on the ABP medium as a cellulase transcriptional activator when expressed in T. orientalis EU7-22. Xylanase activity and $\beta$-glucosidase activity of ace 2 heterogeneous expression strains are improved and total cellulase activity is decreased. The result of qPCR is accordance with enzyme activities. However, the presence of several putative binding sites for Ace2 in a promoter affects enzyme activities significantly and the expression of other regulatory factors in vivo. It provides the potential in the applications for differentiated purposes and its effect on the induced fermentation still requires further studies. This study provides a reference for an indepth study on cellulase transcriptional regulation mechanisms of $T$. orientalis.

\section{Declarations}

\section{Ethical Approval}

No related Ethical issues.

\section{Consent to Participate}

The authors promise that the work described has not been published previously, that it is not under consideration for publication elsewhere, that its publication is approved by all authors and tacitly or explicitly by the responsible authorities where the work was carried out.

\section{Consent to Publish}

The authors promise that if the manuscript is accepted, it will not be published elsewhere in the same form, in English or in any other language, without the written consent of the Publisher. There are no conflicts of interest to declare.

\section{Authors Contributions}

Yuyu Li performed the experiment and wrote the paper.

Yong Xue conceived the idea of the study.

Jian Liu contributed to supervision, fund supporting and revised the manuscript.

Lihui Gan contributed to supervision and analyzed the data.

Minnan Long helped perform the analysis with constructive discussions.

\section{Funding}

This work was supported by National Natural Science Foundation of China (No. 21978249).

\section{Competing Interests}


None.

\section{References}

[1] J. Baeyens, Q. Kang, L. Appels, R. Dewil, Y. Lv, T. Tan, Challenges and opportunities in improving the production of bio-ethanol, Progress in Energy and Combustion Science 47 (2015) 60-88.

[2] H. Fang, L. Xia, High activity cellulase production by recombinant Trichoderma reesei ZU-02 with the enhanced cellobiohydrolase production, Bioresour Technol 144 (2013) 693-7.

[3] R.H. Bischof, J. Ramoni, B. Seiboth, Cellulases and beyond: the first 70 years of the enzyme producer Trichoderma reesei, Microb Cell Fact 15(1) (2016) 106.

[4] J.W. Agger, T. Isaksen, A. Varnai, S. Vidal-Melgosa, W.G. Willats, R. Ludwig, S.J. Horn, V.G. Eijsink, B. Westereng, Discovery of LPMO activity on hemicelluloses shows the importance of oxidative processes in plant cell wall degradation, Proc Natl Acad Sci U S A 111(17) (2014)6287-92.

[5] H. Okada, K.Mori, K. Tada, M. Nogawa, Y. Morikawa, Identification of active site carboxylic residues in Trichoderma reesei endoglucanase Cel12A by site-directed mutagenesis, Journal of Molecular Catalysis B: Enzymatic 10 (2000) 249-255.

[6] L. Wang, X. Lv, Y. Cao, F. Zheng, X. Meng, Y. Shen, G. Chen, W. Liu, W. Zhang, A novel transcriptional regulator RXE1 modulates the essential transactivator XYR1 and cellulase gene expression in Trichoderma reesei, Appl Microbiol Biotechnol 103(11) (2019) 4511-4523.

[7] Y. Xue, J. Han, Y. Li, J. Liu, L. Gan, M. Long, Promoting cellulase and hemicellulase production from Trichoderma orientalis EU7-22 by overexpression of transcription factors Xyr1 and Ace3, Bioresour Technol 296 (2020) 122355.

[8] C. Long, Y.Cheng, X. Wu, J.Liu, M.Long, Cloning and Sequence Analysis of Cellulase Genes from Hypocrea orientalis EU7-22, Biotechnol. Bull. 11(2012) 110-117. .

[9] A. Rassinger, A. Gacek-Matthews, J. Strauss, R.L. Mach, A.R. Mach-Aigner, Truncation of the transcriptional repressor protein Cre1 in Trichoderma reesei Rut-C30 turns it into an activator, Fungal Biol Biotechnol 5 (2018) 15.

[10] C. Long, Y. Cheng, J. Cui, J. Liu, L. Gan, B. Zeng, M. Long, Enhancing Cellulase and Hemicellulase Production in Trichoderma orientalis EU7-22 via Knockout of the creA, Molecular Biotechnology 60(1) (2018) 55-61.

[11] N. Aro, M. Ilmen, A. Saloheimo, M. Penttila, ACEl of Trichoderma reesei is a repressor of cellulase and xylanase expression, Appl Environ Microbiol 69(1) (2003) 56-65. 
[12] J. Zhang, Y. Chen, C. Wu, P. Liu, W. Wang, D. Wei, The transcription factor ACE3 controls cellulase activities and lactose metabolism via two additional regulators in the fungus Trichoderma reesei, $\mathrm{J}$ Biol Chem 294(48) (2019) 18435-18450.

[13] Y. Chen, C. Wu, X. Fan, X. Zhao, X. Zhao, T. Shen, D. Wei, W. Wang, Engineering of Trichoderma reesei for enhanced degradation of lignocellulosic biomass by truncation of the cellulase activator ACE3, Biotechnol Biofuels 13 (2020) 62.

[14] Y. Luo, M. Valkonen, R.E. Jackson, J.M. Palmer, A. Bhalla, I. Nikolaev, M. Saloheimo, M. Ward, Modification of transcriptional factor ACE3 enhances protein production in Trichoderma reesei in the absence of cellulase gene inducer, Biotechnol Biofuels 13 (2020) 137.

[15] A.R. Stricker, K. Grosstessner-Hain, E. Würleitner, R.L. Mach, Xyr1 (Xylanase Regulator 1) Regulates both the Hydrolytic Enzyme System and d-Xylose Metabolism in Hypocrea jecorina, Eukaryotic Cell 5(12) (2006) 2128-2137.

[16] J. Han, Y. Xue, M. Li, Y. Li, J. Liu, L. Gan, M. Long, Effect of VIB Gene on Cellulase Production of Trichoderma orientalis EU7-22, Appl Biochem Biotechnol 191(4) (2020) 1444-1455.

[17] R.B. Todd, A. Andrianopoulos, Evolution of a Fungal Regulatory Gene Family: The Zn(II)2Cys6 Binuclear Cluster DNA Binding Motif, Fungal Genetics and Biology 21 (3) (1997) 388-405.

[18] N. Aro, A. Saloheimo, M. IImen, M. Penttila, ACEll, a novel transcriptional activator involved in regulation of cellulase and xylanase genes of Trichoderma reesei, J Biol Chem 276(26) (2001) 24309-14.

[19] A.R. Stricker, P. Trefflinger, N. Aro, M. Penttila, R.L. Mach, Role of Ace2 (Activator of Cellulases 2) within the xyn2 transcriptosome of Hypocrea jecorina, Fungal Genet Biol 45(4) (2008) 436-45.

[20] L. Xiong, A.K. Kameshwar, X. Chen, Z. Guo, C. Mao, S. Chen, W. Qin, The ACEIl recombinant Trichoderma reesei QM9414 strains with enhanced xylanase production and its applications in production of xylitol from tree barks, Microb Cell Fact 15(1) (2016) 215.

[21] T. Portnoy, A. Margeot, V. Seidl-Seiboth, S. Le Crom, F. Ben Chaabane, R. Linke, B. Seiboth, C.P. Kubicek, Differential regulation of the cellulase transcription factors XYR1, ACE2, and ACE1 in Trichoderma reesei strains producing high and low levels of cellulase, Eukaryot Cell 10(2) (2011) 262-71.

[22] E. Szewczyk, T. Nayak, C.E. Oakley, H. Edgerton, Y. Xiong, N. Taheri-Talesh, S.A. Osmani, B.R. Oakley, Fusion PCR and gene targeting in Aspergillus nidulans, Nature Protocols 1(6) (2006) 3111-3120.

[23] M. Dashtban, M. Maki, K.T. Leung, C. Mao, W. Qin, Cellulase activities in biomass conversion: measurement methods and comparison, Crit Rev Biotechnol 30(4) (2010) 302-9.

[24] K.J. Livak, T.D. Schmittgen, Analysis of Relative Gene Expression Data Using Real-Time Quantitative PCR and the 2- $\triangle \triangle C T$ Method, Methods 25(4) (2001) 402-408. 
[25] J. Li, J. Wang, S. Wang, M. Xing, S. Yu, G. Liu, Achieving efficient protein expression in Trichoderma reesei by using strong constitutive promoters, Microbial Cell Factories 11(1) (2012) 84.

[26] F.Liu, Y.Xue, J.liu, L.Gan, M.Long, ACE3 as a Master Transcriptional Factor Regulates Cellulase and Xylanase Production in Trichoderma orientalis EU7-22, Bioresources 13(3) (2018) 6790-6801.

\section{Figures}

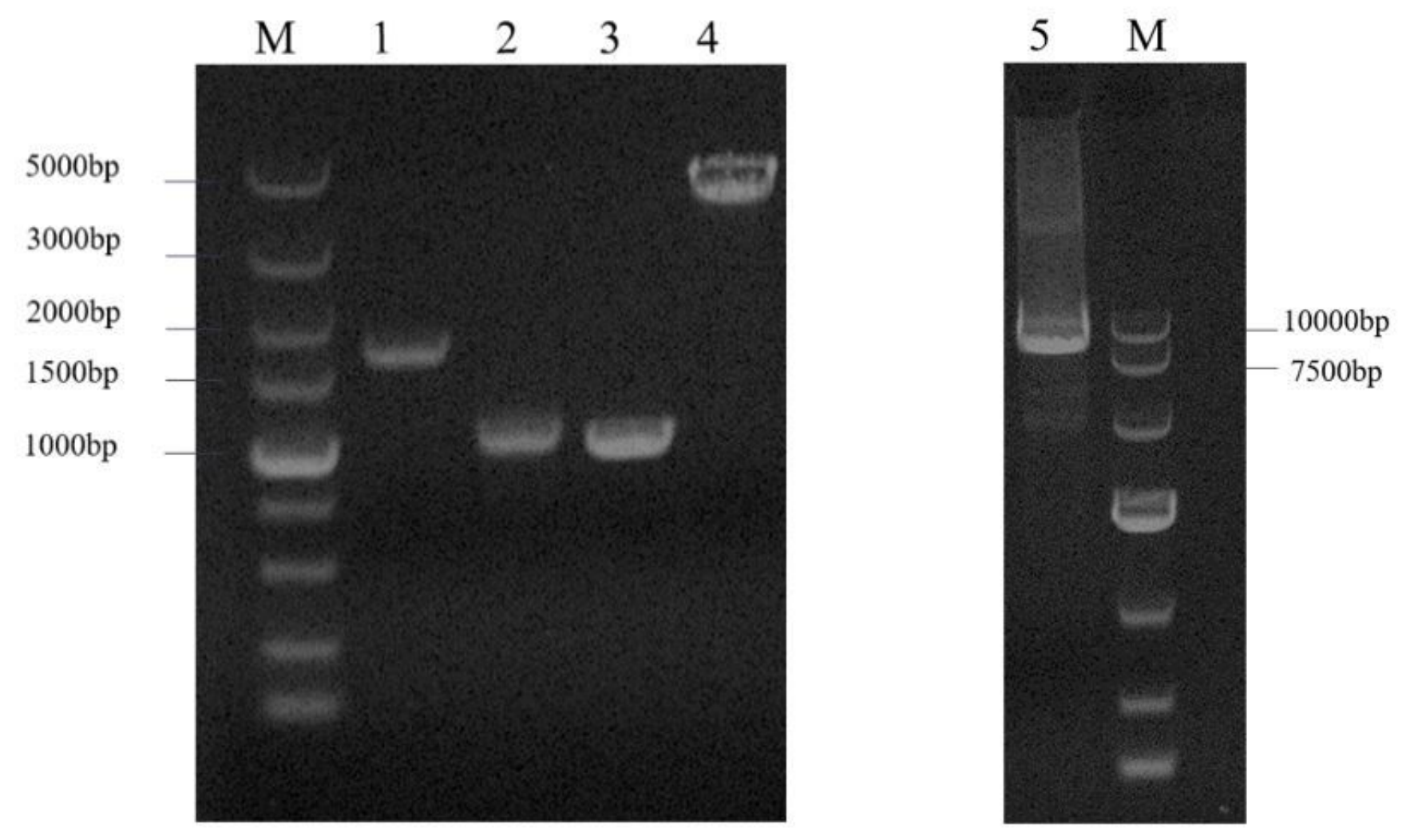

\section{Figure 1}

The electrophoretogram of four segments and integrated segments. M means marker, the 1, 2, 3, 4 and 5 represent the promoter, the terminator of eno, hygromycin resistance cassette and ace 2 expression cassette, respectively. 


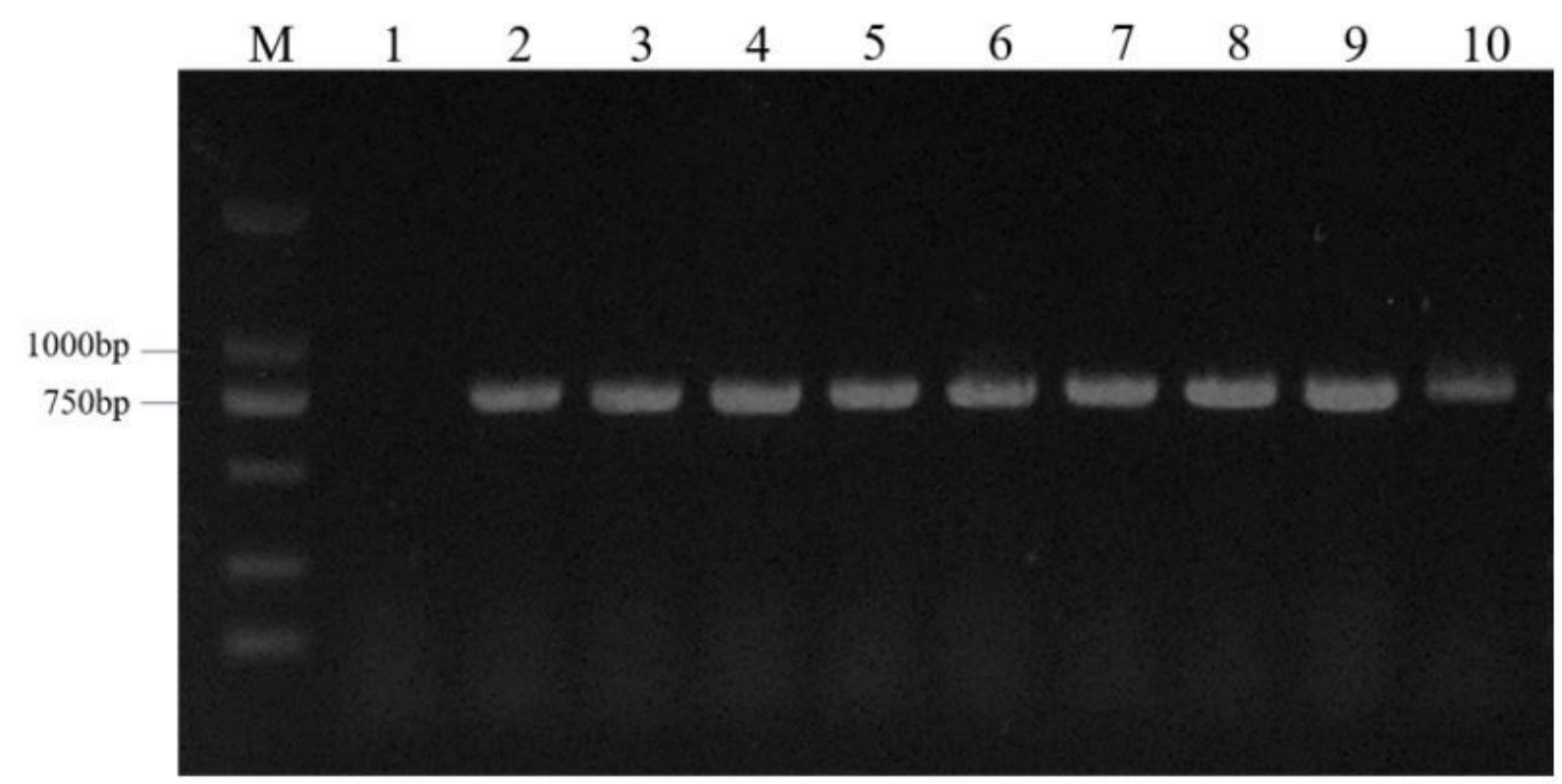

Figure 2

The verification of the transformants by amplifying the partial hph gene. (M) marker, the templates of the PCR are (1) EU7-22, (2) 0-1, (3) 0-2, (4) 0-3, (5) 0-4, (6) 0-5, (7) 1-1, (8) 1-2, (9) 2-9 and (10) 3-1.

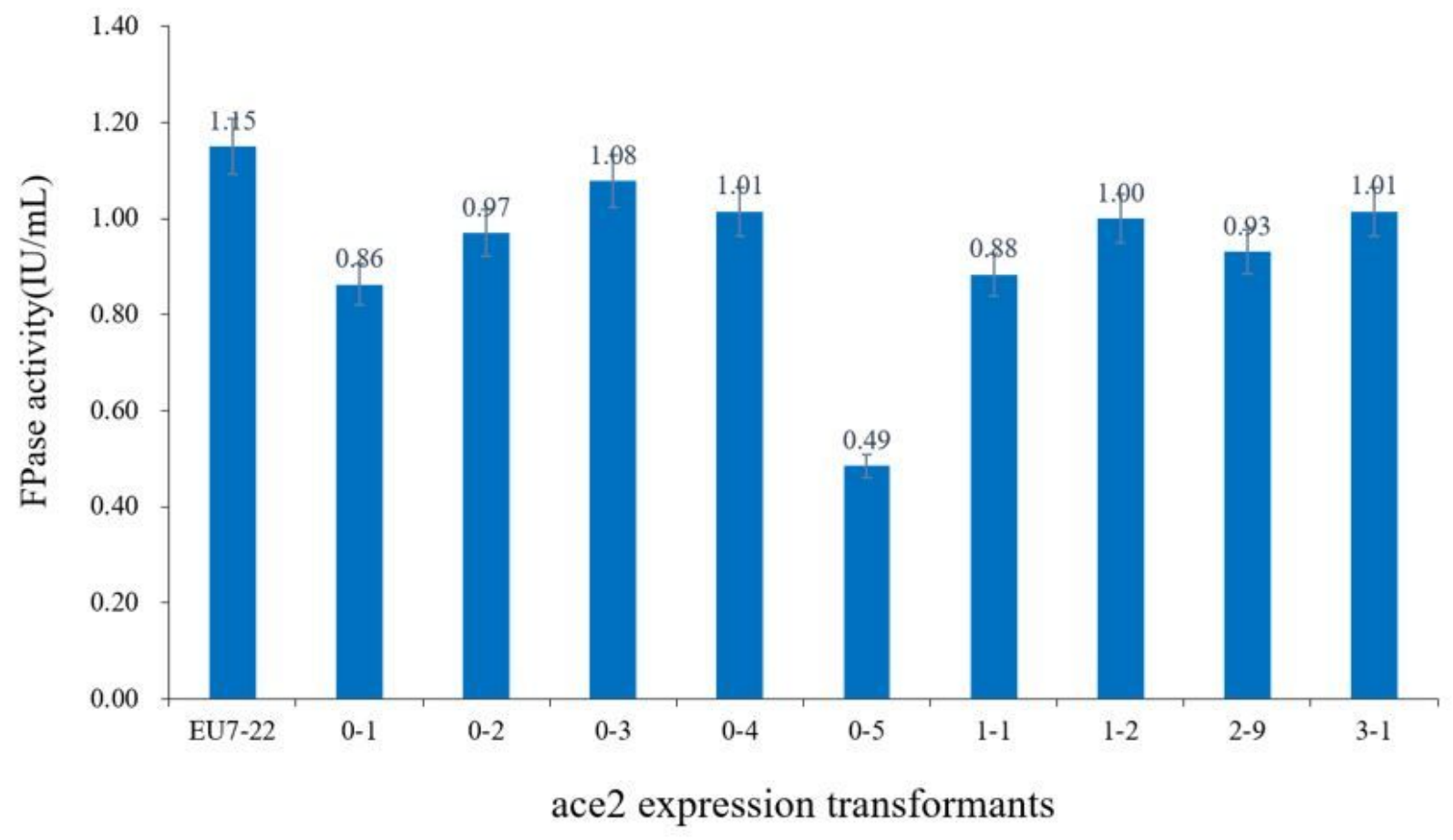

Figure 3

Activities of FPase from EU7-22 and transformants using the inducing medium 

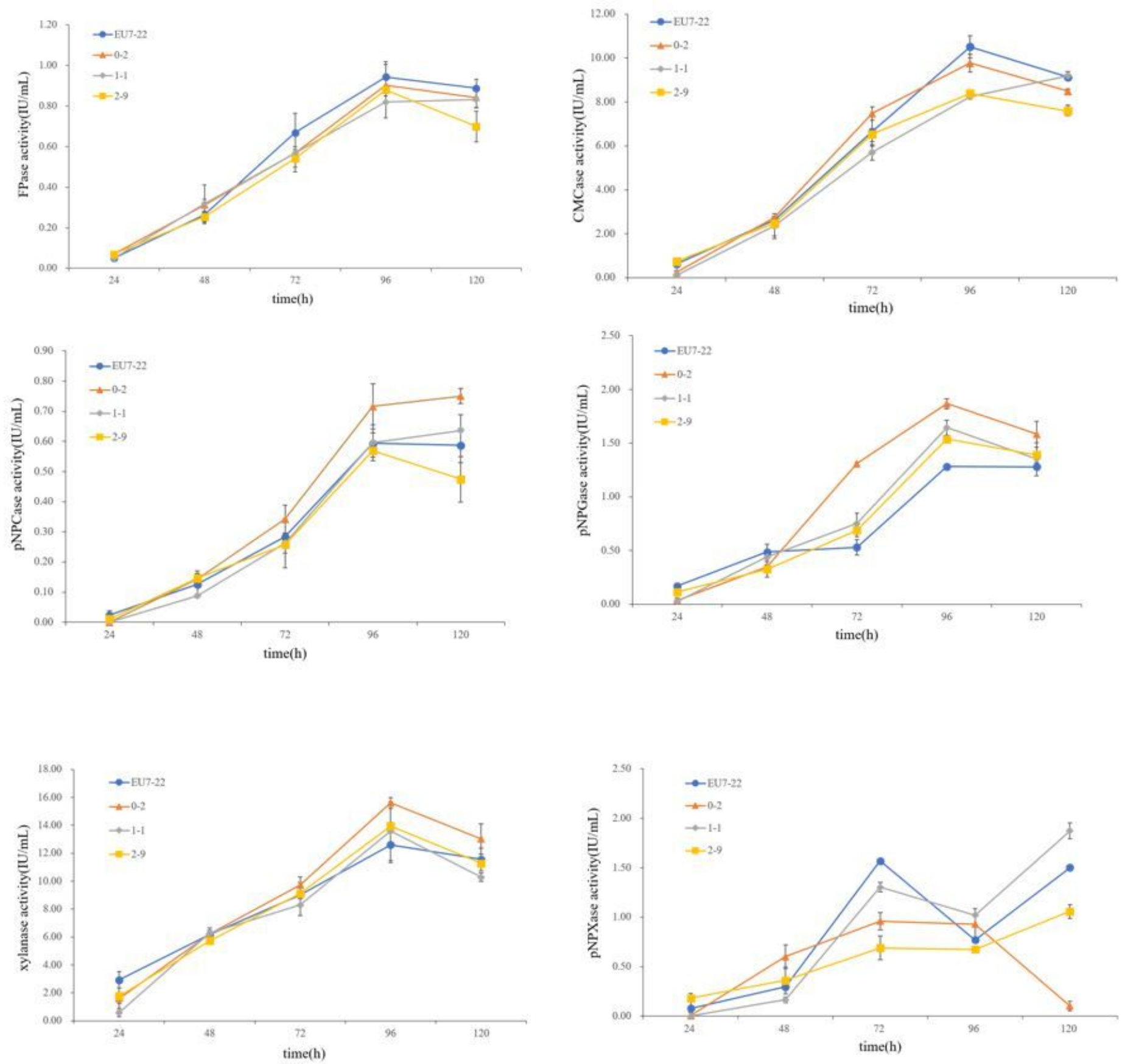

Figure 4

Activities of FPase, CMCase, pNPCase, pNPGase, xylanase and pNPXase of EU7-22 and transformants $(0-2,1-1$ and $2-9)$ using the inducing medium 


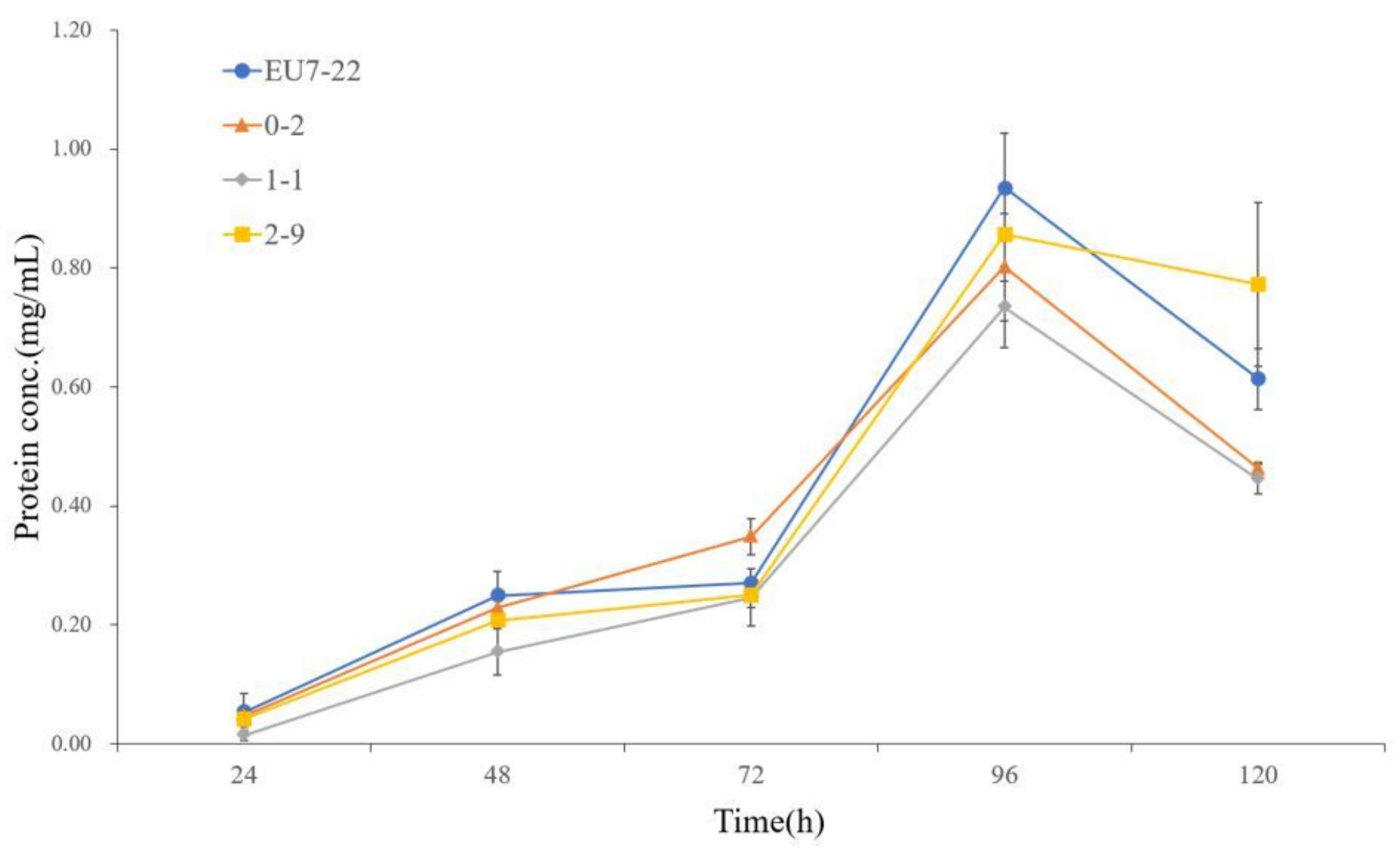

Figure 5

Protein concentrations from T. orientalis EU7-22 and transformants (0-2, 1-1 and 2-9) using the inducing medium 


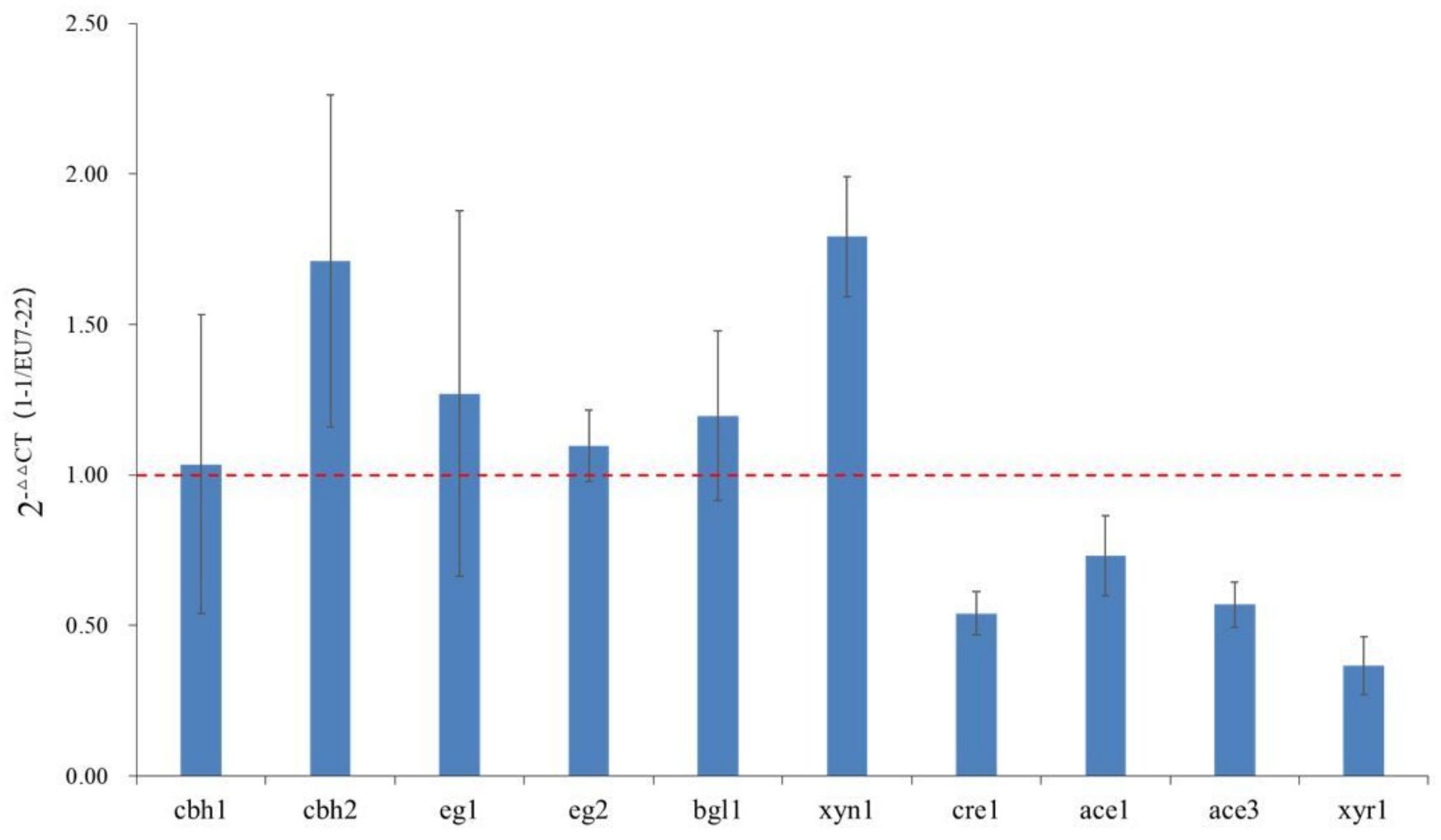

Figure 6

The transcription levels of the main genes at $24 \mathrm{~h}$. 


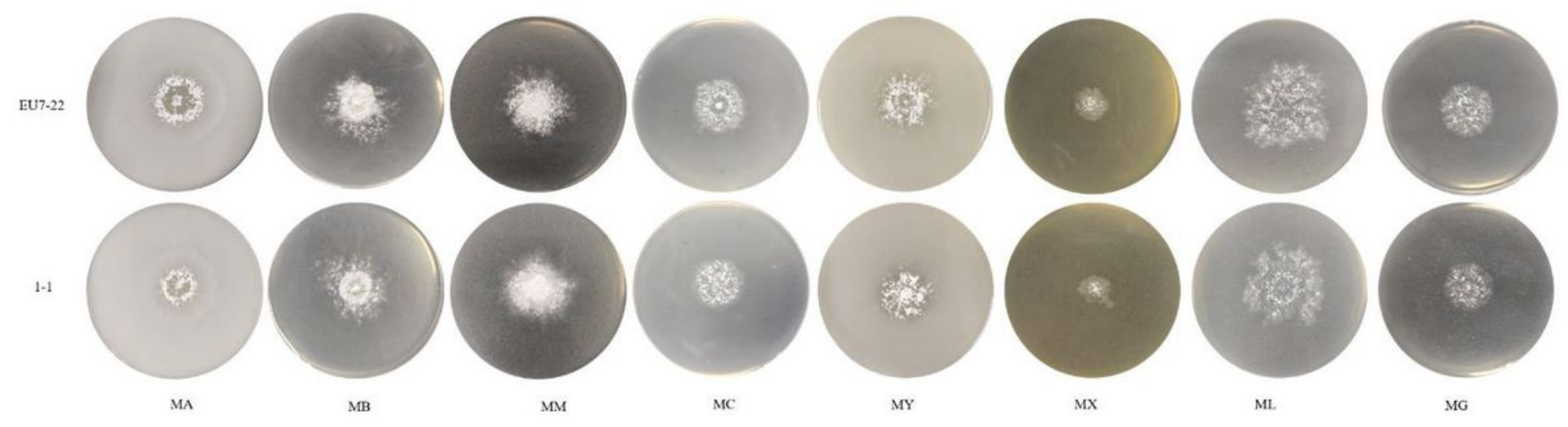

(a)

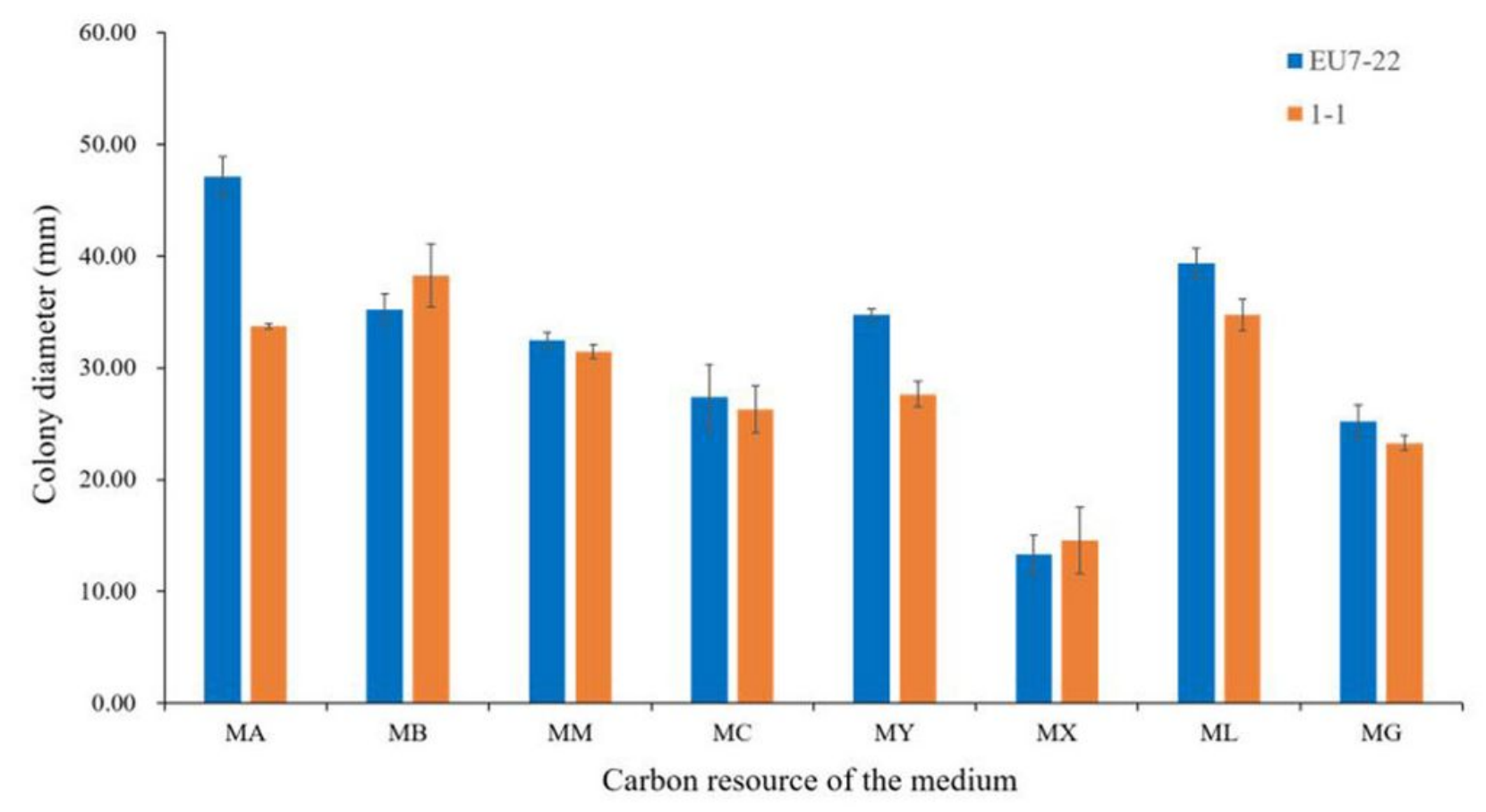

(b)

Figure 7

Colony morphology (a) and spread rate (b) of EU7-22 and 1-1 on different carbon resource medium cultured for $72 \mathrm{~h}$. 